\title{
ON DISCRETE FIELD THEORY PROPERTIES OF THE DIMER AND ISING MODELS AND THEIR CONFORMAL FIELD THEORY LIMITS
}

\author{
IGOR KRIZ, MARTIN LOEBL, AND PETR SOMBERG
}

\begin{abstract}
We study various mathematical aspects of discrete models on graphs, specifically the Dimer and the Ising models. We focus on proving gluing formulas for individual summands of the partition function. We also obtain partial results regarding conjectured limits realized by fermions in rational conformal field theories.
\end{abstract}

\section{INTRODUCTION}

The idea that the dimer and Ising statistical models on finite graphs have, as a limit, the free fermion conformal field theory in dimension 2 and 1, respectively, has become a well established theme in mathematical physics (cf. [29], [8]). Yet, great challenges remain on the road to developing this into a mathematically rigorous theory. A part of the difficulty is in reconciling the languages and concepts of graph theory and conformal field theory: the structure of conformal field theory predicts many features which are not readily visible on graphs. One must develop mechanisms how such features originate on the graph level and will fully manifest themselves in the limit. The purpose of this paper is, in some sense, to begin this investigation in earnest. We record some of the phenomena which need to modelled. We also obtain concrete results as first steps in the desired direction.

While conformal field theory was long considered a part of the domain of physics, rigorous mathematical approaches now do exist. Perhaps most appealing is the "naive" approach due to Graeme Segal [31, which asks for a Hilbert space $H$, and for each Riemann surface $\Sigma$ with analytically parametrized boundary components, an element $U(\Sigma)$ (defined up to scalar multiple) of the Hilbert tensor product of copies of $H$ and its dual indexed by the boundary components (depending on orientation), satisfying appropriate "gluing axioms". The paper [31] is just an outline, and details have been since filled in, in part by Kriz and his co-authors [22, 18, 11].

A competing approach, starting with the notion of vertex algebra, was started by Borcherds [3] and Frenkel-Lepowsky-Meurman [14 (see also Beilinson-Drinfeld [2]). This approach models only a part of the gluing structure postulated by Segal, but has the advantage that a vertex algebra is a completely algebraic concept, which does not require analysis. A vertex algebra, however, models only a part of conformal field theory, the "chiral 0-sector". An

1991 Mathematics Subject Classification. 82B20, 52C99, 05C30, 81T40, 57M15.

Key words and phrases. Dimer model, Ising model, Conformal field theory, Gluing formulas, Critical embeddings and limits.

I. Kriz was supported by NSF grant DMS 1102614, P. Somberg would like to acknowledge a support of the research project MSM 0021620839 and the grant GA ČR 201/08/0397. 
extension of the approach which models a whole conformal field theory, was developed by Huang and Lepowsky [19], but it does involve analysis, although not Hilbert spaces. Efforts to model the entire conformal field theory as a completely algebraic concept are underway [17, 23].

The mathematically richest case of conformal field theory is a rational conformal field theory, when there is a finite set of labels $\Lambda$ with an involution (?)* and the Hilbert space breaks up as

$$
H=\bigoplus_{\lambda \in \Lambda} H_{\lambda} \hat{\otimes} \bar{H}_{\lambda^{*}}
$$

The Hilbert spaces $H_{\lambda}$ are called chiral sectors and are subject to additional axioms. Parallel to the formula (11), there is a formula on graded dimensions, the partition function formula (formula (45) below). A major advantage of the vertex algebra approach is that it predicts from a vertex algebra alone (i.e. algebraic data) when we get a rational conformal field theory, and gives an algebraic formula for its sectors (see also [2]).

The free fermion is now completely described mathematically as a conformal field theory both in the Segal and vertex algebra formalisms (see [31, 22, 19]). Yet, the theory is more subtle than one might think. We describe some of the issues involved in Section 66 below. For one thing, a rational conformal field theory in the precise sense outlined above actually describes the bosonisation of the free fermion. There is a variant of the above description, called a fermionic rational conformal field theory, which is more closely related to the topic of this paper. It is actually the fermionic rational conformal field theory analogue of the partition function formula (formula (54), and its analogues for higher genus) we are interested in. In the case of the free fermion, this formula (10) is precisely a sum over spin structures of a Riemann surface of genus $g$, with a factor $1 / 2^{g}$, which matches the formula on graph dimer partition function found by Cimasoni and Reshetikhin [4].

The present paper is mainly about graphs. How can we model the concepts mentioned discretely? Fortunately, great strides have already been made. Mercat and Kenyon [20, 21] defined critically embedded graphs on Riemann surfaces, which give discrete models the conformal structure. A basic quantum field theory-like gluing property of the dimer model was observed by Cimasoni and Reshetikhyn [5].

A large part of the present paper (Sections 2, 3) consists of examining the gluing property in more detail, both for the Ising and dimer models. Our main new result in this direction is proving a gluing formula not just for the entire partition function, but for its summands, i.e. discrete analogues of (10), (54). More precisely, we consider arbitrary labellings, not only critical ones; this generality, the formula is actually related to quantum field theory (not conformal field theory). The conformal case, however, is the one we need to consider for taking scaling limits. For simplicity, we restrict to the case of a double torus being cut into two surfaces of genus 1 , but it is straightforward to generalize the formula to more general cuts along separating curves.

Our gluing formula is substantially more difficult to obtain than the formula of [5]. Instead of cutting along a system of edges of a graph embedded into a Riemann surface by a separating curve, we consider a separating curve containing vertices only of the graph. To obtain a 
cutting (gluing) formula for partition functions, we must perform a certain graph-theoretical constrution on the graphs resulting from the cut (introducing new subgraphs which we call core in the case of the dimer and target in the case of the Ising model). Our main gluing theorems are Theorems 10 and 6 of Section 3 .

To work our way closer toward a limit formula, we need to understand how gluing works mathematically in the fermion conformal field theory. This is done in Section 4. Even though there are both mathematical and physical approaches (e.g. [34, 22, 9]), as far as we know, there is no treatment in the literature which would include a mathematically precise definition of the data at a closed Riemann surface in terms of the Dirac operator, and the related cases of gluing. We write the Dirac operator on a Riemann surface in a conformally invariant form, and write down a definition and gluing properties of its determinant with respect to gluing along curves with antiperiodic spin structure. (The determinant is the square of the Pfaffian: we restrict to the case of the determinant because it is much simpler technically.)

A rigorous discrete-to-continuous limit formula for one of the chiral partition functions of the chiral fermion on a torus is the topic of Section 5. Previous work on this was done by Cohn, Kenyon and Propp who work out a formula in the case of rectangular dominoes [6] (in fact, in the case of a parallelogram, it was worked out already by Kasteleyn [20, see also Ferdinand [10]). A heuristic approach in a more general situation is done in [8]. A general obstacle to deal with, which we haven't mentioned above, is the conformal anomaly, namely the fact that the elements $U(\Sigma)$ are defined only up to scalar multiple. (In the chiral sector, this propagates into the elaborate structure of a modular functor, some of which is described in Section 6.) To deal with this difficulty, one encounters a phenomenon in nearly all discreteto-continuous limits in physics: regularization. Typically, one encounters a limit which does not exist, and is "regularized" by replacing it with a somewhat different but compellingly similar expression. In the present setting, we prove for arbitrary periodic critical embedding in a torus that the limit does not exist, and can be regularized by replacing, at one point, a set of parameters by their absolute values, after which the CFT limit is obtained.

There is yet another complication in a rigorous approach to discrete-to-continuous limits in the case of the Ising model, which does not arise in the case of the dimer model: while in the case of the dimer model, the limit theory is the 2-dimensional fermion CFT, for the Ising model, the limit theory should be the 1-dimensional fermion [29], which, despite similar terminology, is considerably more subtle, and involves a construction of P. Deligne using super-central simple algebras over $\mathbb{C}$. Whether natural discrete versions of these concepts exist is an open problem, which we discuss at the end of Section 6 .

\section{Discrete Gluing: The Set-uP}

Let $G=(V(G), E(G))$ be a finite unoriented graph consisting of vertices $V(G)$ and edges $E(G)$, in which loop-edges and multiple edges are allowed. We say that the subset $E^{\prime} \subset E(G)$ is even if the graph $\left(V(G), E^{\prime}\right)$ has even degree (icluding degree zero) at each vertex. We say that $M \subset E(G)$ is a perfect matching or dimer arrangement if the graph $(V(G), M)$ has degree one at each vertex. Let $\mathcal{E}(G)$ denote the set of all even subgraphs of $G$, and let $\mathcal{P}(G)$ denote the set of all perfect matchings (dimers) of $G$. 
We assume that an indeterminate $x_{e}$ is associated with each edge $e \in E(G)$, and $x_{e}$ may be evaluated in complex numbers. We define the generating functions for even subsets of $E(G)$ resp. for perfect matchings, $\mathcal{E}_{G}$ resp. $\mathcal{P}_{G}$, as elements of $\mathbb{Z}\left[\left(x_{e}\right)_{e \in E(G)}\right]$ :

$$
\begin{aligned}
& \mathcal{E}_{G}(x)=\sum_{E^{\prime} \in \mathcal{E}(G)} \prod_{e \in E^{\prime}} x_{e}, \\
& \mathcal{P}_{G}(x)=\sum_{M \in \mathcal{P}(G)} \prod_{e \in M} x_{e} .
\end{aligned}
$$

Polynomial $\mathcal{P}_{G}(x)$ is also known as the dimer partition function of graph $G$. Knowing the polynomial $\mathcal{E}_{G}$ is equivalent to knowing the partition function $Z_{G}^{\text {Ising }}$ of the Ising model on the graph $G$, defined by

$$
Z_{G}^{\text {Ising }}(\beta)=\left.Z_{G}^{\text {Ising }}(x)\right|_{x_{e}:=e^{\beta J_{e}} \forall e \in E(G)}
$$

where the $J_{e}(e \in E(G))$ are weights (coupling constants) associated with the edges of the graph $G$, the parameter $\beta$ is the inverse temperature, and

$$
Z_{G}^{\text {Ising }}(x)=\sum_{\sigma: V(G) \rightarrow\{1,-1\}} \prod_{e=\{u, v\} \in E(G)} x_{e}^{\sigma(u) \sigma(v)} .
$$

The theorem of van der Waerden [33] states that $Z_{G}^{\text {Ising }}(x)$ is equivalent to $\mathcal{E}_{G}(x)$ up to a change of variables and multiplication by a constant factor:

$$
Z_{G}^{\text {Ising }}(x)=\left.2^{|V(G)|}\left(\prod_{e \in E(G)} \frac{x_{e}+x_{e}^{-1}}{2}\right) \mathcal{E}_{G}(z)\right|_{z_{e}:=\frac{x_{e}-x_{e}^{-1}}{x_{e}+x_{e}^{-1}}}
$$

Let us assume the vertices of $G$ are numbered from 1 to $n$. If $D$ is an orientation of $G$, we denote by $A(G, D)$ the skew-symmetric adjacency matrix of $D$ defined as follows: The diagonal entries of $A(G, D)$ are zero, and the off-diagonal entries are

$$
A(G, D)_{i j}=\sum \pm x_{e},
$$

where the sum is over all edges $e$ connecting vertices $i$ and $j$, and the sign in front of $x_{e}$ is 1 if $e$ is oriented from $i$ to $j$ in the orientation $D$, and -1 otherwise. As is well-known, the Pfaffian of this matrix counts perfect matchings of $G$ with signs:

$$
\text { Pfaf } A(G, D)=\sum_{M \in \mathcal{P}(G)} \operatorname{sign}(M, D) \prod_{e \in M} x_{e},
$$

where $\operatorname{sign}(M, D)= \pm 1$. We use this as the definition of the sign of a perfect matching $M$ with respect to an orientation $D$. It is well known that we have for any pair of perfect matchings $M, N$

$$
\operatorname{sign}(M, D) \cdot \operatorname{sign}(N, D)=(-1) \text { the number of D-even cycles in } M \cup N .
$$

An even-length cycle is $D$-even if it has an even number of directed edges in agreement with one way of traversal.

We denote the polynomial Pfaf $A(G, D) \in \mathbb{Z}\left[\left(x_{e}\right)_{e \in E(G)}\right]$ by $F_{D}(x)$ and call it the Pfaffian associated to the orientation $D$. The following result is well-known: 
Theorem 1 (Kasteleyn [20], Galluccio-Loebl [15], Tesler [32], Cimasoni-Reshetikhin [4]). If $G$ embeds into an orientable surface of genus $g$, then there exist $4^{g}$ orientations $D_{i}(i=$ $\left.1, \ldots, 4^{g}\right)$ of $G$ such that the perfect matching polynomial $\mathcal{P}_{G}(x)$ can be expressed as a linear combination of the Pfaffian polynomials $F_{D_{i}}(x)$.

We call this expression the Arf-invariant formula, as it is based on a property of Arf invariant for quadratic forms in characteristic two. As far as we know, the relationship with Arf invariant was first observed in [4].

Let the graph $G$ be embedded in a closed Riemann surface $X$ of genus $g$. We denote $H:=H_{1}\left(X, \mathbb{F}_{2}\right)$ the first homology group of $X$ with coefficients in the field $\mathbb{F}_{2}$. Recall that $H$ carries a non-degenerate skew-symmetric bilinear form called $(\bmod 2)$ intersection form and denoted by ' $'$. Quadratic form on $(H, \cdot)$ associated to '.' is a function $q: H \rightarrow \mathbb{F}_{2}$ fulfilling

$$
q(x+y)=q(x)+q(y)+x \cdot y
$$

for all $x, y \in H$.

We denote $Q$ the set of quadratic forms on $H$ over $\mathbb{F}_{2}$, its cardinality being $4^{g}$.

Each quadratic form $q \in Q$ determines the signed generating function of even subsets of $G$ by the formula

$$
\mathcal{E}_{G, q}(x)=\sum_{E^{\prime} \in \mathcal{E}(G)}(-1)^{q\left(\left[E^{\prime}\right]\right)} \prod_{e \in E^{\prime}} x_{e} .
$$

The generating function $\mathcal{E}_{G}(x)$ of the even subsets of edges admits analogous Arf invariant formula, where $\mathcal{E}_{G}(x)$ is expressed as a linear combination of its signed modifications $\mathcal{E}_{G, q}(x), q \in Q$, see [25].

Analogously, partition function of the free fermion conformal field theory in (spacetime) dimension 2 on a closed Riemann surface $X$ of genus $g$ can be expressed as a linear combination of $2^{2 g}$ functional Pfaffians of the Dirac operators, each of them corresponding to a Spin-structure on $X$. From a physical perspective, this was noted in e.g. [1], and rigorous mathematical approaches now also exist (see Sections 4, 6 below).

Quite recently an extensive effort was invested in the understanding of the Dimer and Ising models, their criticality resp. asymptotic behaviour in terms of the conformal invariance and quantum field theory (conformal field theory in the critical case) of the free fermion and related theories (see e.g. [1], 77, [34, [5], 25], 8]). We continue in this effort and show that the dimer and the Ising partition functions share with the partition function of the free fermion a basic gluing property of their signed modifications.

We present the gluing formulas for finite graphs embedded in double torus $T^{2}=T \# T$, regarded as a connected sum of two 2-tori (elliptic curves) $T$. The restriction is done for the sake of simplicity, i.e. analogous formulas can be proven for closed Riemann surfaces of any genus.

2.1. Exterior algebra of gluing variables. Let us consider a graph $G$ embedded in a double torus $T^{2}$. We denote by $C$ a simple closed curve (a cycle) on $T^{2}$ with the following properties satisfied:

(1) $T^{2} \backslash C$ has two connected components, each of which is a torus with a disc bounded by $C$ cut out. 
(2) $C$ intersects embedded graph $G$ in an even number of vertices and in no inner point of an edge.

(3) $G$ has an even number of vertices in each component of $T^{2} \backslash C$.

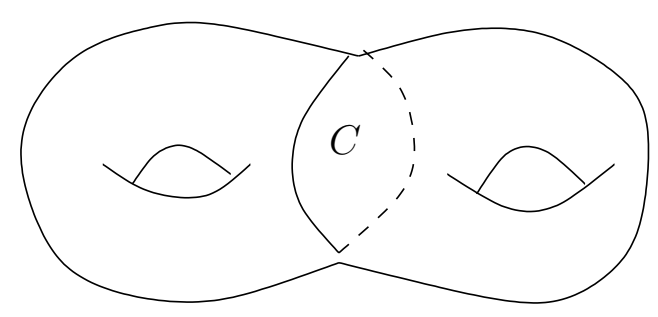

Figure 1. The double torus

Let $v^{1}, \ldots, v^{k}, k$ even, be the vertices of $G$ on $C$ ordered along $C$. We consider the exterior algebra of $2 k$ gluing variables

$$
e_{1}^{1}<e_{2}^{1}<\ldots<e_{1}^{k}<e_{2}^{k}
$$

ordered according to the orientation of $C$ and the choice of a vertex on $C$. Then the vector space on the basis $B$ consisting of all monomials

$$
e_{\epsilon_{1}}^{i_{1}} e_{\epsilon_{2}}^{i_{2}} \ldots e_{\epsilon_{\ell}}^{i_{\ell}}
$$

with

$$
e_{\epsilon_{1}}^{i_{1}}<e_{\epsilon_{2}}^{i_{2}}<\ldots<e_{\epsilon_{\ell}}^{i_{\ell}}
$$

is naturally identified with the exterior algebra

$$
\Lambda\left(e_{1}^{1}, e_{2}^{1}, \ldots, e_{1}^{k}, e_{2}^{k}\right)
$$

The dual exterior algebra

$$
\Lambda\left(e_{1}^{1}, e_{2}^{1}, \ldots, e_{1}^{k}, e_{2}^{k}\right)^{*}=\Lambda\left(e_{2}^{k *}, e_{1}^{k *}, \ldots, e_{2}^{1 *}, e_{1}^{1 *}\right)
$$

is naturally identified with the vector space on the basis $B^{*}$ consisting of all monomials

$$
e_{\epsilon_{\ell}}^{i_{\ell} *} \ldots e_{\epsilon_{1}}^{i_{1 *}} .
$$

Let us consider $Y \in \Lambda\left(e_{1}^{1}, e_{2}^{1}, \ldots, e_{1}^{k}, e_{2}^{k}\right)$ written as a linear combination of basis elements $Y=\sum_{b \in B} y_{b} b$, and similarly $Z \in \Lambda\left(e_{1}^{1}, e_{2}^{1}, \ldots, e_{1}^{k}, e_{2}^{k}\right)^{*}$ written as $Z=\sum_{b^{\prime} \in B^{*}} z_{b^{\prime}} b^{\prime}$. Then the scalar product $\langle Y, Z\rangle$ is defined by

$$
<Y, Z>=\sum_{\left(b, b^{\prime}\right)}<y_{b} b, z_{b^{\prime}} b^{\prime}>,
$$

where $<y_{b} b, z_{b^{\prime}} b^{\prime}>$ is written in the following way. Let $b=e_{\epsilon_{1}}^{i_{1}} e_{\epsilon_{2}}^{i_{2}} \ldots e_{\epsilon_{\ell}}^{i_{\ell}}$ and $b^{\prime}=e_{\alpha_{m}}^{j_{m} *} \ldots e_{\alpha_{1}}^{j_{1} *}$. Then $<b, b^{\prime}>=0$ unless $m=\ell$ and $\epsilon_{p}=\alpha_{p}, i_{p}=j_{p}$ for each $p$, in which case $<y_{b} b, z_{b^{\prime}} b^{\prime}>=$ $y_{b} z_{b^{\prime}}$.

We first illustrate the gluing by writing down a gluing formula for the unsigned partition function $\mathcal{E}_{G}(x)$. This was done in a different way for the dimer partition function $\mathcal{P}_{G}(x)$ in [5]. 
2.2. Discrete unsigned gluing. We denote by $G_{1}, G_{2}$ the subgraphs of $G$ supported on connected components of $T^{2} \backslash C$ along with the vertices of $G$ supported on $C$. Hence, if $v$ is a vertex of $G$ embedded in $C$ then the set $B_{v}$ of the edges incident with $v$ is partitioned into two sets $B_{1}(v), B_{2}(v)$, the first belonging to $G_{1}$ and the second to $G_{2}$.

We construct an auxiliary graph, called the target, as follows. For each $i=1, \ldots, k$, let us introduce the path $L(i)$ of length 2 containing the vertices $v_{1}^{i}, v_{2}^{i}, v_{3}^{i}$ and the edges $\left\{v_{3}^{i} v_{2}^{i}\right\},\left\{v_{2}^{i} v_{1}^{i}\right\}$. The target $S$ is obtained as the union of paths $L(i), i=1, \ldots, k$ by identifying all $k$ vertices $v_{3}^{i}, i=1, \ldots, k$ into one single vertex (see Figure 2 for the target $S$ ). The target $S$ allows to construct the graph $G_{j}^{S}$ from $G_{j}$ and $S$ by identifying, for each $i=1, \ldots, k$ the vertices $v^{i}$ and $v_{1}^{i}$.

The edge-variables of $S$ are the gluing variables. We denote by $y_{1 v_{3}^{i} v_{2}^{i}}=e_{1}^{i}, y_{1 v_{2}^{i} v_{1}^{i}}=e_{2}^{i}$ the gluing variables in $G_{1}^{S}$ and by $y_{2 v_{3}^{i} v_{2}^{i}}=e_{2}^{i *}, y_{2 v_{2}^{i} v_{1}^{i}}=e_{1}^{i *}$ the gluing variables in $G_{2}^{S}$.

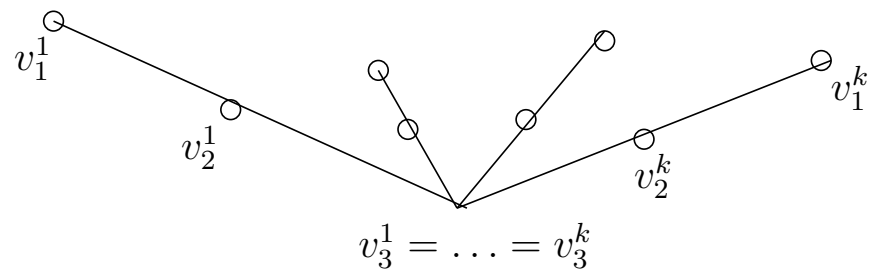

Figure 2. The target $S$

The vector $y_{1}$ of variables of $G_{1}^{S}$ consists of $\left(x_{e}\right)_{e \in E(G)}$ and the gluing variables $\left(e_{1}^{1}, e_{2}^{1}, \ldots, e_{1}^{k}, e_{2}^{k}\right)$. The vector $y_{2}$ of variables of $G_{2}^{S}$ consists of $\left(x_{e}\right)_{e \in E(G)}$ and the gluing variables $\left(e_{2}^{k *}, e_{1}^{k *}, \ldots, e_{2}^{1 *}, e_{1}^{1 *}\right)$. The following theorem simply follows from the definition of scalar product in the exterior algebra.

\section{Theorem 2.}

$$
\mathcal{E}_{G}(x)=<\mathcal{E}_{G_{1}^{S}}\left(y_{1}\right), \mathcal{E}_{G_{2}^{S}}\left(y_{2}\right)>.
$$

\section{Discrete Signed GLUING}

Many results in this section exploit the structure of $g$-graph, extensively used and explained in detail in, e.g. [24, [25] and references therein.

Definition 3. The highway surface $\mathbb{S}_{g}$ consists of a base polygon $R_{0}$ and collection of bridges $R_{1}, \ldots, R_{2 g}$, where

(1) $R_{0}$ is a convex $4 g$-gon with vertices $a_{1}, \ldots, a_{4 g}$ numbered clockwise,

(2) Each $R_{2 i-1}$ is a rectangle with vertices $x(i, 1), \ldots, x(i, 4)$ numbered clockwise, and glued to $R_{0}$ in a way that its edge $[x(i, 1), x(i, 2)]$ is identified with the edge $\left[a_{4(i-1)+1}, a_{4(i-1)+2}\right]$ and the edge $[x(i, 3), x(i, 4)]$ is identified with the edge $\left[a_{4(i-1)+3}, a_{4(i-1)+4}\right]$,

(3) Each $R_{2 i}$ is a rectangle with vertices $y(i, 1), \ldots, y(i, 4)$ numbered clockwise. It is glued to $R_{0}$ so that its edge $[y(i, 1), y(i, 2)]$ is identified with the edge $\left[a_{4(i-1)+2}, a_{4(i-1)+3}\right]$ and the edge $[y(i, 3), y(i, 4)]$ is identified with the edge $\left[a_{4(i-1)+4}, a_{4(i-1)+5}\right]$. Notice that indices are considered modulo $4 \mathrm{~g}$.

There is an orientation-preserving map $\Phi$ of $\mathbb{S}_{g}$ into the plane $\mathbb{R}^{2}$, which is an immersion up to the images of the bridges $R_{2 i}$ and $R_{2 i-1}$ intersecting in a square for each $i=1, \ldots g$. 
Let us assume the graph $G$ is embedded into a closed orientable surface $X$ of genus $g$. The combinatorial model of $X$ is $\mathbb{S}_{g}$ unified with an additional disk $R_{\infty}$ glued to the boundary of $\mathbb{S}_{g}$. By an isotopy property of the embedding, we may assume that $G$ does not meet the disk $R_{\infty}$ and moreover, all vertices of $G$ lie in the interior of $R_{0}$. We may also assume that the intersection of $G$ with any of the rectangular bridges $R_{i}$ consists of disjoint straight lines connecting the two sides of $R_{i}$ glued to the base polygon $R_{0}$. This assumption is not really necessary, but makes all considerations below more transparent. The composition of the embedding of $G$ into $\mathbb{S}_{g}$ with the immersion $\Phi$ yields a drawing $\varphi$ of $G$ in the plane $\mathbb{R}^{2}$. A planar drawing of $G$ obtained in this way will be called special. Observe that double points of a special drawing can only come from the intersection of the images of bridges under the immersion $\Phi$ of $\mathbb{S}_{g}$ into the plane. Thus every double point of a special drawing lies in one of the squares $\Phi\left(R_{2 i}\right) \cap \Phi\left(R_{2 i-1}\right)$.

In what follows we employ this machinary to produce gluing formulas.

3.1. The Ising model. As for the notation and basic results, we follow [25].

Definition 4. Let $G$ be a graph embedded in $\mathbb{S}_{g}$ and let e be an edge of $G$. By definition, the embedding of e intersects each bridge $R_{i}$ in a collection of disjoint straight lines, whose number is denoted $r_{i}(e)$. For a subset $A$ of edges of $G$ we denote $r(A)$ the vector of length $2 g$, whose $i$-th component is $r(A)_{i}=\sum_{e \in A} r_{i}(e)$.

We observe that two even subsets of edges $A, B$ belong to the same homology class in $H$ if and only if $r(A)=r(B)(\bmod 2)$. We pick a basis $a_{1}, b_{1}, \ldots, a_{g}, b_{g}$ of $H$, where $a_{i}, b_{i}$ correspond to the even subsets $A_{i}, B_{i}$ satisfying $r\left(A_{i}\right)_{2 i-1}=r\left(B_{i}\right)_{2 i}=1$ and all remaining components of $r\left(A_{i}\right)$ and $r\left(B_{i}\right)$ are zero. Note that each quadratic form in $Q$ is uniquelly determined by its values on any basis of the underlying vector space.

The following Theorem is a basic result of ([25]).

Theorem 5. Let $G$ be a graph embedded in a closed Riemann surface $X$ of genus $g$ and let $q$ be a quadratic form on the homology group $H$. Let $E^{\prime}$ be an even subset of edges of $G$ and let $r=r\left(E^{\prime}\right)$. Then

$$
(-1)^{q\left(\left[E^{\prime}\right]\right)}=(-1)^{m\left(E^{\prime}\right)}
$$

where

$$
m\left(E^{\prime}\right)=\sum_{i=1}^{g} r_{2 i-1} r_{2 i}+r_{2 i-1} q\left(a_{i}\right)+r_{2 i} q\left(b_{i}\right) .
$$

The importance of Theorem 5 is related to the following observation. Let us consider an embedding of $G$ in the highway surface $\mathbb{S}_{2}$, for simplicity considered of genus $g=2$. A quadratic form $q \in Q$ naturally determines two quadratic forms $q_{1}, q_{2}$ on the torus by $q_{1}\left(a_{1}\right)=q\left(a_{1}\right), q_{1}\left(b_{1}\right)=q\left(b_{1}\right)$ resp. $q_{2}\left(a_{1}\right)=q\left(a_{2}\right), q_{2}\left(b_{1}\right)=q\left(b_{2}\right)$ together with trivial values on the other basis elements not written explicitly.

The signed version of Theorem 2 is of the following form.

Theorem 6. We have

$$
\mathcal{E}(G, q, x)=<\mathcal{E}\left(G_{1}^{S}, q_{1}, y_{1}\right), \mathcal{E}\left(G_{2}^{S}, q_{2}, y_{2}\right)>.
$$

Proof: Using Theorem 2 the proof is easily reduced to 
Claim. Let $E^{\prime}$ be an even subset of edges of $G$. For $i=1,2$, there is exactly one extension $E_{i}^{\prime}$ of $E^{\prime} \cap G_{i}$ to an even subset of $G_{i}^{S}$ by edges in the target. Moreover, for arbitrary $q \in Q$,

$$
(-1)^{q\left(\left[E^{\prime}\right]\right)}=(-1)^{q_{1}\left(\left[E_{1}^{\prime}\right]\right)}(-1)^{q_{2}\left(\left[E_{2}^{\prime}\right]\right)} .
$$

The Claim follows directly from Theorem 5 and definitions of $q_{1}, q_{2}$. This finishes the proof of Theorem 6 .

3.2. The dimer model. In this subsection we present a gluing formula for the signed dimer partition function of a graph $G$ embedded in the closed Riemann surface of genus 2. Analogously to the case of the Ising partition function, we first introduce an auxiliary graph called the core and denoted $S^{\prime}$. The construction of the core follows the procedure of reduction of the Ising model to the dimer model, see [13].

We recall that in Subsection 2.2 we denoted by $G_{1}, G_{2}$ the subgraphs of $G$ supported on connected components of $T^{2} \backslash C$ together with the vertices of $G$ supported on $C$. If $v$ is a vertex of $G$ embedded in $C$ then the set $B_{v}$ of the edges incident with $v$ is partitioned into two sets $B_{1}(v), B_{2}(v)$, the first belonging to $G_{1}$ and the second to $G_{2}$. For each $i=1, \ldots, k, L(i)$ denotes the path of length 2 consisting of the vertices $v_{1}^{i}, v_{2}^{i}, v_{3}^{i}$ and the edges $\left\{v_{3}^{i} v_{2}^{i}\right\},\left\{v_{2}^{i} v_{1}^{i}\right\}$.

To construct the core $S^{\prime}$, we first consider the path consisting of $6 k$ new vertices $u_{1}, \ldots, u_{6 k}$ and edges $\left\{u_{i}, u_{i+1}\right\}, i=1, \ldots 6 k-1$. Then we add the edges $\left\{u_{3 j-2}, u_{3 j}\right\}, j=1, \ldots, 2 k$ and identify, for $i=1, \ldots, k$, the vertex $u_{6 i-4}$ with $v_{3}^{i}$ (see Figure 3 for an orientation of the core).

Finally, for $j=1,2$ we construct the graphs $G_{j}^{S^{\prime}}$ from the union of $G_{j}$ and $S^{\prime}$ by identifying, for each $i=1, \ldots, k$ the vertices $v^{i}$ and $v_{1}^{i}$.

The relevance of the core stems from the following observation, see e.g. [13].

Observation 7. Let $A \subset\left\{v_{1}^{i}, i=1, \ldots, k\right\}$. Then $S^{\prime}$ has a matching completely covering all vertices of $S^{\prime} \backslash A$ if and only if $|A|$ is even. Moreover, if $|A|$ is even, the matching completely covering $S^{\prime} \backslash A$ is uniquelly determined.

The edge-variables of the paths $L(i)$ are, as in the construction of the target, the gluing variables. For $i=1, \ldots, k$, we denote by $z_{1 v_{3}^{i} v_{2}^{i}}=e_{1}^{i}, z_{1 v_{2}^{i} v_{1}^{i}}=e_{2}^{i}$ the gluing variables in $G_{1}^{S^{\prime}}$ resp. $z_{2 v_{3}^{i} v_{2}^{i}}=e_{2}^{i *}, z_{2 v_{2}^{i} v_{1}^{i}}=e_{1}^{i *}$ the gluing variables in $G_{2}^{S^{\prime}}$.

We let vector $z_{1}$ of variables of $G_{1}^{S^{\prime}}$ consist of $\left(x_{e}\right)_{e \in E(G)}$, the gluing variables $\left(e_{1}^{1}, e_{2}^{1}, \ldots, e_{1}^{k}, e_{2}^{k}\right)$ and the remaining edge variables be set to 1 . We let vector $z_{2}$ of the variables of $G_{2}^{S^{\prime}}$ consist of $\left(x_{e}\right)_{e \in E(G)}$, the gluing variables $\left(e_{2}^{k *}, e_{1}^{k *}, \ldots, e_{2}^{1 *}, e_{1}^{1 *}\right)$, and the remaining edge variables be set to 1 .

We are now ready to discuss gluing formula for unsigned partition function. The following Theorem simply follows from the definition of the trace in the exterior algebra, using Observation 7 .

\section{Theorem 8.}

$$
\mathcal{P}_{G}(x)=<\mathcal{P}_{G_{1}^{S^{\prime}}}\left(z_{1}\right), \mathcal{P}_{G_{2}^{S^{\prime}}}\left(z_{2}\right)>
$$

In the next step we consider the signed case. We first modify $G$ by adding the edges $\left\{v^{i}, v^{i+1}\right\}, i=1, \ldots, k-1$ and let the variables associated to these edges be equal to 0 . Hence, adding this set of edges does not change the signed dimer partition function of $G$. Let us denote the modified graph by $G^{\prime}$. 


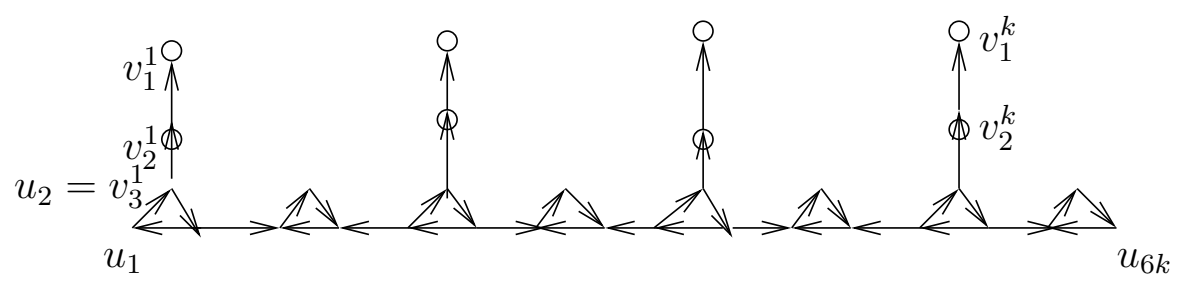

Figure 3 . The orientation of the core $S^{\prime}$

A result of [32] (see also recent [24]) can be summarized, in the case of the double torus, as follows:

Theorem 9. Let $G$ be a graph embedded in a highway surface $\mathbb{S}_{2}$. Let $D$ be an orientation of $G$ so that for each bridge $R$, the immersion of $R_{0} \cup R$ into the plane has the property that each inner face has an odd number of edges oriented clokwise in D. Let $M$ be a perfect matching of $G$. Then

$$
\operatorname{sign}(M, D)=(-1)^{c(M)},
$$

where $c(M)$ denotes the number of self-intersections among edges of $M$ inside the special drawing of $G$ in the plane.

Let $G$ be the graph embedded in the double torus $T^{2}$. We consider the embedding of $G^{\prime}$ in the highway surface $\mathbb{S}_{2}$ determined by an embedding of $G$ in the closed Riemann surface $T^{2}$ of genus 2 . Note that the new edges attached among the vertices of $C$ form a chord of the base polygon $R_{0}$, which separates the two pairs of bridges. We recall that $v^{1}, \ldots, v^{k}$ is the order of the vertices of $G$ on $C$ along this chord, and that $k$ is even.

It is not difficult to observe that there is an orientation $D_{0}$ of $G^{\prime}$ fulfilling the properties of Theorem 9, where the orientations of the edges $\left\{v^{i}, v^{i+1}\right\}, i=1, \ldots, k-1$, form a directed path of odd length from $v^{1}$ to $v^{k}$.

Let $D$ be an orientation of $G$. We denote by $D_{1}$ the restriction of $D$ to $G_{1}$ and by $D_{2}$ the restriction of $D$ to $G_{2}$. We consider the orientation of the core $S^{\prime}$, indicated on Figure 3. By slight abuse of notation we denote it also by $S^{\prime}$. If $D$ is an orientation of $G$ then we denote by $D_{1}^{S^{\prime}}, D_{2}^{S^{\prime}}$ the orientations of $G_{1}^{S^{\prime}}, G_{2}^{S^{\prime}}$ induced by $D$ and $S^{\prime}$.

Theorem 10. Let $D$ be an orientation of $G$. Then

$$
F_{D}(x)=<F_{D_{1}^{S^{\prime}}}\left(z_{1}\right), F_{D_{2}^{S^{\prime}}}\left(z_{2}\right)>.
$$

Proof: Let $M$ be a perfect matching of $G$. It follows from Observation 7 that for $i=1,2$, there is exactly one extension $M_{i}$ of $M \cap G_{i}$ to perfect matching of $G_{i}^{S^{\prime}}$ by the core edges. Theorem 10 follows from Theorem 8 and

Claim.

$$
\operatorname{sign}(M, D)=\operatorname{sign}\left(M_{1}, D_{1}^{S^{\prime}}\right) \cdot \operatorname{sign}\left(M_{2}, D_{2}^{S^{\prime}}\right) .
$$

We first show that the Claim holds for the orientation $D_{0}$ introduced above. Since $M$ is a subset of edges of $G$, we consider $D_{0}$ restricted to $G$ only. We have by Theorem 9

$$
\operatorname{sign}\left(M, D_{0}\right)=(-1)^{c(M)},
$$


where $c(M)$ denotes the number of self-intersections among the edges of $M$ in the special drawing of $G^{\prime}$ in the plane. From the construction of $G^{\prime}$ in Subsection 3.2 we have

$$
c(M)=c_{1}(M)+c_{2}(M),
$$

where $c_{1}(M)$ denotes the number of self-intersections among the edges of $M$ that belong to the first pair of the bridges of the highway surface $\mathbb{S}_{2}$, and $c_{2}(M)$ denotes the number of self-intersections among the edges of $M$ that belong to the second pair of the bridges of the highway surface $\mathbb{S}_{2}$.

Finally, the orientations $D_{1}^{S^{\prime}}, D_{2}^{S^{\prime}}$ are constructed from $D_{0}$ in such a way that they also satisfy the properties required by Theorem 9. Hence

$$
\begin{aligned}
& \operatorname{sign}\left(M_{1}, D_{1}^{S^{\prime}}\right)=(-1)^{c_{1}\left(M_{1}\right)}, \\
& \operatorname{sign}\left(M_{2}, D_{2}^{S^{\prime}}\right)=(-1)^{c_{2}\left(M_{2}\right)} .
\end{aligned}
$$

Summarising, the Claim holds for the orientation $D_{0}$. An arbitrary orientation $D$ of $G$ is obtained from $D_{0}$ by reversing orientation in a subset $S(D)$ of the directed edges of $D_{0}$, so

$$
\operatorname{sign}(M, D)=\operatorname{sign}\left(M, D_{0}\right)(-1)^{|S(D) \cap M|}
$$

and

$$
\begin{aligned}
& \operatorname{sign}\left(M_{1}, D_{1}^{S^{\prime}}\right)=\operatorname{sign}\left(M_{1}, D_{1}^{S^{\prime}}\right)(-1)^{\left|S(D) \cap M_{1}\right|}, \\
& \operatorname{sign}\left(M_{2}, D_{2}^{S^{\prime}}\right)=\operatorname{sign}\left(M_{2}, D_{2}^{S^{\prime}}\right)(-1)^{\left|S(D) \cap M_{2}\right|} .
\end{aligned}
$$

This finishes the proof of the last Claim and at the same time of Theorem 10.

\section{FERMions ON COMPACT SURFACES I}

In this section, we present some facts about the free fermion conformal field theory in the context of the desired limit from the discrete to the continuous case. From the physics point of view, this material is more than two decades old, see e.g. [1, 34]. A mathematical approach was outlined in [31], and further developed in [22, 18]. Nevertheless, the mathematical literature as it stands is deficient in an important point (explained below), which happens to be the key to addressing the limit questions we are interested in. Treating this gap is the main purpose of the present section.

The point is that in the mathematical treatments of CFT, closed Riemann surfaces (or compact Riemann surfaces without boundary) play a special role, and a technical complication. It is a common technique, in fact, to consider only (compact) Riemann surfaces without closed connected components, and argue that data at closed surfaces can be recovered by cutting out a holomorphic disk, and giving compatible isomorphisms of the data as the disk varies. This is the approach taken in [31, 22].

For our purposes, however, this is unsatisfactory. The reason is that from the physical point of view, the case of a closed surface is in fact more fundamental ([34]), because it is where contact with quantum field theory is made. The data we are supposed to have in this case is the determinant (resp. Pfaffian) of the Dirac operator, which is analogous to the discrete data we studied in sections 2, 3, 
The purpose of this section, then, is to explain how to define the determinant precisely, and to describe gluing from the data on a surface with boundary as described in [31, 22] to the Dirac operator data in the closed surface case. For simplicity, we treat only the determinant, which corresponds to the free fermion of spacetime dimension 2, and not the Pfaffian, which is more complicated. Also for reasons of simplicity, we discuss only antiperiodic boundary conditions. The role of all spin structures will be reviewed in more detail in Section 6 below. A discrete analogue of the 'boundary to no boundary' gluing case will be, in fact, exhibited in Section 5 below, where we give one prototype of a rigorous regularized CFT limit computation.

Let $\Sigma$ be a compact Riemann surface. A spin structure on $\Sigma$ can be identified with a choice of a holomorphic line bundle $\underline{\Omega^{1 / 2}}$ on $\Sigma$ and an isomorphism

$$
\underline{\Omega^{1 / 2}} \otimes \underline{\Omega^{1 / 2}} \cong \underline{\Omega^{1}} \text {. }
$$

(We identify a holomorphic bundle with its sheaf of sections $\underline{M}$; we denote by $M$ the space of global holomorphic sections; $\underline{M}_{s m}, M_{s m}$ denotes the corresponding notions in the smooth category.) The bundles of spinors on $\Sigma$ are $\underline{\Delta^{-}}=\underline{\Omega^{-1 / 2}}$ and $\underline{\Delta^{-}}=\underline{\Omega^{+1 / 2}}$.

The Dirac operator

$$
\Delta_{s m}^{-} \rightarrow \Delta_{s m}^{+}
$$

ordinarily depends on metric (i.e. is only defined on a spin Riemann manifold), which is undesirable for the purposes of CFT. However, a modified conformally invariant construction can be obtained as follows: We define a pairing

$$
\not D: \Omega_{s m}^{1 / 2} \otimes_{\mathbb{C}} \Omega_{s m}^{1 / 2} \rightarrow \mathbb{C}
$$

in a local holomorphic coordinate $z$ as follows:

$$
f d z^{1 / 2} \otimes g d z^{1 / 2} \mapsto \int_{\Sigma} \frac{\partial f}{\partial \bar{z}} g d z d \bar{z}
$$

To see that (9) is invariant under holomorphic coordinate change, let $z=z(y)$. Using the $y$ coordinate, we compute

$$
\begin{gathered}
f d z^{1 / 2} \otimes g d z^{1 / 2}=f\left(\frac{d z}{d y}\right)^{1 / 2} d y^{1 / 2} \otimes g\left(\frac{d z}{d y}\right)^{1 / 2} d y^{1 / 2} \mapsto \\
\mapsto \int \frac{\partial f}{\partial \bar{y}} g \frac{d z}{d y} d y d \bar{y}=\int \frac{\partial f}{\partial \bar{z}} g \frac{d z}{d y} \frac{d \bar{z}}{d \bar{y}} d y d \bar{y}=\int \frac{\partial f}{\partial \bar{z}} g d z d \bar{z}
\end{gathered}
$$

Also note that by Stokes' theorem, $\not D$ is an antisymmetric pairing, since

$$
\left(\frac{\partial f}{\partial \bar{z}} g+f \frac{\partial g}{\partial \bar{z}}\right) d z d \bar{z}=d(f g d z)
$$

One defines (up to scalar multiple) the partition function of the 1-dimensional chiral fermion on $\Sigma$ as

$$
p f(\not D) \text {. }
$$

The partition function of a fermion with both chiralities is

$$
\frac{1}{2^{g}} \sum_{\sigma} \alpha(\sigma) p f\left(\begin{array}{cc}
\not D_{\sigma} & 0 \\
0 & \widetilde{D D}_{\sigma}
\end{array}\right)
$$


where $\widetilde{D D}$ is the construction analogous to $\not D$ with "holomorphic" replaced by "anti-holomorphic" and $\alpha(\sigma)$ is the Arf invariant.

To define the Pfaffian, one proceeds as follows. For an antisymmetric Fredholm operator

$$
\Phi: H \rightarrow H^{*}
$$

where $H$ is a Hilbert space (we are not in this situation, but may achieve it by suitable Hilbert-completion), there is a Pfaffian line $\operatorname{Pf}(\Phi)$ (well defined up to canonical isomorphism), and a Pfaffian element

$$
p f(\Phi) \in \operatorname{Pf}(\Phi)
$$

(see [30], Chapter 12). For simpicity, we will describe only the square of (11),

$$
\operatorname{det}(\Phi) \in \operatorname{Det}(\Phi) \text { for } \Phi: H_{1} \rightarrow H_{2} \text { Fredholm of index } 0 .
$$

Choose an invertible operator $J: H_{1} \rightarrow H_{2}$ such that

$$
T=J-\Phi
$$

is trace class. (For any compact operator $K: H_{1} \rightarrow H_{2}$, there exist unique numbers $s_{1} \geq s_{2} \geq$ $\ldots \geq 0, \lim s_{n}=0$ and bases $\left(e_{n}\right),\left(f_{n}\right)$ of $H_{1}, H_{2}$, respectively, such that $K\left(e_{n}\right)=s_{n} f_{n}$. The numbers $s_{n}$ are called singular values. $K$ is trace class (resp. Hilbert-Schmidt) if $\sum\left|s_{n}\right|<\infty$ $\left(\right.$ resp. $\left.\sum\left(s_{n}\right)^{2}<\infty\right)$.)

Now one defines

$$
\operatorname{Det}(\Phi):=\langle(J)\rangle
$$

(=the line with free formal generator $(J)$ ), and

$$
\operatorname{det}(\Phi):=\operatorname{det}\left(I-T J^{-1}\right) \cdot(J) \in \operatorname{Det}(\Phi)
$$

(the determinant is defined as a number because $T J^{-1}$ is trace class). For another choice

$$
\Phi=J^{\prime}-T^{\prime}
$$

with $J^{\prime}$ invertible and $T^{\prime}$ trace class, we have

$$
J^{\prime} J^{-1}=I+\left(T^{\prime}-T\right) J^{-1}
$$

so the canonical iso

$$
\left\langle\left(J^{\prime}\right)\right\rangle \rightarrow\langle(J)\rangle
$$

is

$$
\left(J^{\prime}\right) \mapsto(J) \cdot \operatorname{det}\left(I+\left(T^{\prime}-T\right) J^{-1}\right)
$$

One has $\operatorname{det}(\Phi)=0$ unless $\Phi$ is invertible. To define $\operatorname{det}(\Phi)$ as a number, one must specify a generator of $\operatorname{Det}(\Phi)$. This is called regularization.

Let us turn back to Pfaffians. In our situation, this amounts to picking a section of the pullback of the bundle $P f\left(\not D_{\Sigma}\right)$ on the Teichmüller space. This can be done using the structure of CFT.

For a Riemann surface $\Sigma$ with spin structure with analytically parametrized boundary such that the induced spin structure on each boundary component is antiperiodic, there is a non-degenerate quadratic form on the space $\Omega_{\partial \Sigma}^{1 / 2}$ of complex-valued $1 / 2$-forms on the boundary given by

$$
B(\omega, \eta)=\int_{\partial \Sigma} \omega \eta
$$


For each boundary component, 1/2-forms which extend to the unit disk holomorphically form a maximal isotropic subspace $\left(\Omega_{S^{1}}^{1 / 2}\right)^{-}$of $\left(\Omega_{S^{1}}^{1 / 2}\right)$. One defines

$$
\mathcal{H}_{S^{1}}=\left(\Lambda\left(\Omega_{s^{1}}^{1 / 2}\right)^{-}\right)^{\wedge}
$$

(here $?^{\wedge}$ denotes Hilbert completion,) and

$$
\mathcal{H}_{\partial \Sigma}:=\widehat{\bigotimes}\left(\mathcal{H}_{S^{1}}\right)^{*} \hat{\otimes} \widehat{\bigotimes}_{S^{1}}
$$

where $\hat{\otimes}$ denotes Hilbert tensor product, and the tensor products on the right hand side are over boundary components with inbound resp. outbound orientation (the orientation of the boundary component of the standard disk $D$ parametrized by the identity is outbound).

In fact, more explanation is in order: In the present situation, note that the symmetric bilinear form $B$ together with the Real structure (complex conjugation) specifies an inner product on $\Omega_{S^{1}}^{1 / 2}$ (hence on $\Omega_{\partial \Sigma}^{1 / 2}$ ) which is the specific one we use in forming the Hilbert completion, (and hence also defining, say, the inner product on $\Lambda\left(\left(\Omega_{S^{1}}^{1 / 2}\right)^{-}\right)$etc.

Now the inclusion

$$
\left(\Omega_{\Sigma}^{1 / 2}\right)^{\wedge} \subset\left(\Omega_{\partial \Sigma}^{1 / 2}\right)^{\wedge}
$$

where the second map is orthogonal projection is an inclusion of a maximal isotropic subspace which is restricted in the sense that its orthogonal projection to $\left(\left(\Omega_{\partial \Sigma}^{1 / 2}\right)^{-}\right)^{\wedge}$ is Fredholm, while the orthogonal projection to the orthogonal complement $\left(\left(\Omega_{\partial \Sigma}^{1 / 2}\right)^{+}\right)^{\wedge}$ is Hilbert-Schmidt.

In this situation, $\Omega_{\Sigma}^{1 / 2}$ specifies a line $\operatorname{Pf}\left(\Omega_{\Sigma}^{1 / 2}\right)$ and a linear map

$$
\operatorname{Pf}\left(\Omega_{\Sigma}^{1 / 2}\right) \rightarrow \mathcal{H}_{\partial \Sigma}
$$

Now when $\Sigma$ is a closed Riemann surface with spin structure, define

$$
\operatorname{Pf}\left(\Omega_{\Sigma}^{1 / 2}\right):=\operatorname{Pf}\left(\not D_{\Sigma}\right) \text {. }
$$

Lemma 11. Let $\check{\Sigma}$ be a Riemann surface obtained from $\Sigma$ by gluing some inbound boundary components to outbound boundary components in a way so as to preserve parametrization. Then this data specifies an isomorphism

$$
\operatorname{Pf}\left(\Omega_{\Sigma}^{1 / 2}\right) \stackrel{\cong}{\stackrel{\iota}{\longrightarrow}} \operatorname{Pf}\left(\Omega_{\check{\Sigma}}^{1 / 2}\right)
$$

and a commutative diagram where the rows are given by CFT structure

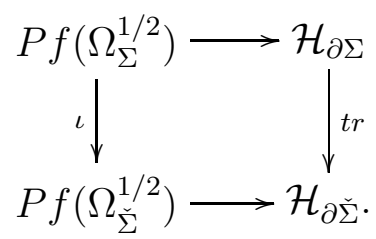

(In the case when $\check{\Sigma}$ is closed, the bottom right corner is $\mathbb{C}$, and the bottom row is defined to be $p f(\not D)^{-1}$.)

We will show only the construction of the map (17) in the case when $\check{\Sigma}$ is closed (i.e. where the definitions of the two Pfaffian lines are different). Also, for simplicity, we will only treat the square, i.e. the analogous construction for determinant lines: the construction for Pfaffians is similar but more elaborate. 
Denote by $\left(\Omega_{0}^{1 / 2}\right)_{\Sigma}$ the subspace of the space of smooth sections $\omega$ of $\left(\underline{\Omega}_{s m}^{1 / 2}\right)_{\Sigma}$ with the property that for any two boundary components in $\partial \Sigma$ which are to be glued in $\check{\Sigma}$,

$$
\left.\bar{\partial} \omega\right|_{c_{1}}=\left.\bar{\partial} \omega\right|_{c_{2}} \text {. }
$$

Denote the image of $\partial \Sigma$ in $\check{\Sigma}$ by $\partial \Sigma / 2$. Then we have a commutative diagram

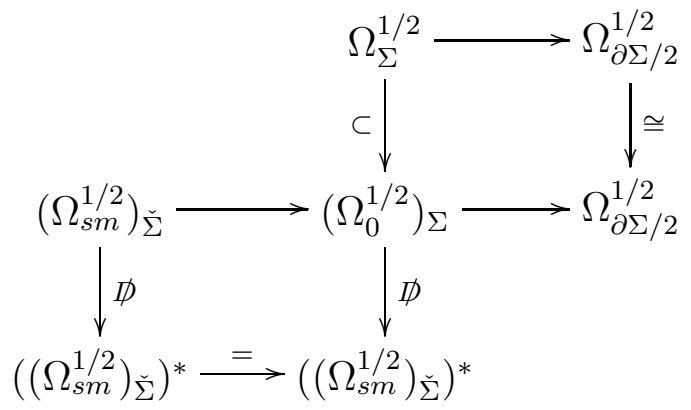

where all rows and columns are short exact with the exception of the top row and left column, which are Fredholm (to reduce clutter, we omit the symbols for Hilbert completions). The top row differs from (14) by a trace class operator (note: ultimately, this difference gives rise to the eta-factor!), so it suffices to prove that for any diagram of Hilbert spaces and bounded operators

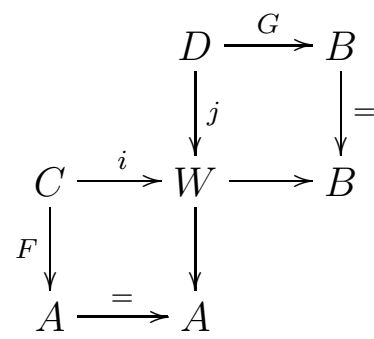

where all the rows and columns are short exact except $F$ and $G$ which are Fredholm, we have a canonical isomorphism

$$
\operatorname{Det}(F) \cong \operatorname{Det}(G)
$$

But note that in (20), we may write $B=W / C, A=W / D$, so both sides are obviously canonically isomorphic to

$$
\operatorname{Det}(i \oplus j: C \oplus D \rightarrow W) .
$$

The commutativity of the square (18) is proved in [22].

Now for the case $\Sigma=A_{q}$ (the standard annulus in $\mathbb{C}$ with boundary components parametrized by $z, q z$ and antiperiodic spin structure), the Lemma specifies a canonical isomorphism

$$
\operatorname{Pf}\left(\Omega_{A_{q}}^{1 / 2}\right) \otimes \operatorname{Pf}\left(\Omega_{D}^{1 / 2}\right) \rightarrow \operatorname{Pf}\left(\Omega_{D}^{1 / 2}\right),
$$

which specifies a canonical element

$$
\iota_{q} \in \operatorname{Pf}\left(\Omega_{A_{q}}^{1 / 2}\right) .
$$

Considering the elliptic curve $\check{\Sigma}=E_{q}$ over $\mathbb{C}$, the image

$$
\xi_{q}:=\iota\left(\iota_{q}\right) \in \operatorname{Pf}\left(\Omega_{E_{q}}^{1 / 2}\right)
$$


is the desired regularization. With respect to this regularization, the partition function is one of the two Jacobi theta functions involving odd powers of $q^{1 / 2}$ (depending on the spin of the gluing), times the factor

$$
\prod_{n>0}\left(1-q^{n}\right)=\eta(\tau) q^{1 / 24}, q=e^{2 \pi i \tau} .
$$

The remaining Arf invariant 0 theta function is not accessible in this way, but may be obtained by cutting around a different simple curve in $E_{q}$ of anti-periodic spin structure; after multiplying by the factor $q^{-1 / 24}$, it is related to the remaining Arf invariant 0 partition functions by a modular transformation. The Arf invariant 1 partition function is 0 .

In the next section, we will see that the partition function of the chiral fermion is, in a special case where the boundary can be easily modelled discretely, a "regularized limit" of partition functions of dimer models on critically embedded graphs, using a discrete analogue of Lemma 11 .

In Section 2 Theorem 8, we exhibited a discrete analogue of Lemma 11 for arbitrary (not necessarily critically embedded) finite graphs, embedded into a Riemann surface of genus 2.

\section{A Limit FORMUla FOR THE DiscRete Dimer MODEL ON A TORUS}

We use here the setup of Kenyon [21]. Consider a bipartite planar graph with vertices colored black and white. The set of black (resp. white) vertices is denoted by $B$ resp. $W$. A critical embedding into a plane is such that the vertices of each face lie on a circle, and all these circles have the same radius. One defines a square matrix $\bar{\partial}$ with rows and columns indexed by $B \cup W$ as follows: If $v_{1}, v_{2}$ are not adjacent, then $\bar{\partial}\left(v_{1}, v_{2}\right)=0$. If $w$ and $b$ are adjacent vertices, $w$ being white and $b$ being black, then

$$
\bar{\partial}(w, b)=-\bar{\partial}(b, w)
$$

is the complex number of length given by twice the distance of the center of the circle circumscribed around (either) face containing $b, w$ to the mid-point between $b$ and $w$, and direction pointing from $w$ to $b$. This is the total Kasteleyn matrix, which we denote by

$$
\left(\begin{array}{rr}
0 & K \\
-K & 0
\end{array}\right)
$$

where the top (resp. bottom) set of rows corresponds to white (resp. black) vertices. We refer to $K$ as the (discrete) chiral Kasteleyn matrix. (In (25), our convention differs from [21], where the matrix is symmetrical; however, antisymmetric matrices fit better with Pfaffians.)

In view of the discrete field theory of Cimasoni-Reshetikhin, the material of Section 4 above suggests a formula of the following form: Let $\Gamma$ be a graph critically embedded into $\mathbb{C} / \mathbb{Z}\{1, \tau\}$ such that $[0,1]$ is antiperiodic. Let $b$ be a black vertex and let $f_{b}(z)$ be the corresponding discrete exponential. Then one should have for the chiral Kasteleyn matrix $K$,

$$
\operatorname{Det}(K) \sim \prod_{\substack{z: f_{b+1}(z)=-f_{b}(z) \\ 16}}\left(1 \pm f_{b+\tau}(z)\right)
$$


where the sign is the spin structure on $[0, \tau]$.

This formula is further motivated by the result of Mercat [26] that exponentials generate additively the vector space of discrete holomorphic functions; rewritten in the basis of discrete exponentials, the chiral Kasteleyn matrix becomes diagonal, so its determinant becomes the product of the diagonal terms, analogously to formula (26) in Section 4 above. There are however two caveats to applying Mercat's theorem: first of all, Mercat is considering a slightly different setup, not assuming that the graph $\Gamma$ is bipartite. He works with tilings by rhomboids; the graph made by the edges of the rhomboids is naturally bipartite, with the vertices of the graphs $\Gamma, \Gamma^{*}$ being the vertices of the two different colors. Second, Mercat's result applies to tilings of simply connected subsets of $\mathbb{C}$, and he does not specify how many discrete exponentials are needed to get a basis of the space of discrete holomorphic functions; in order for the formula (26) to be strictly correct, we would have to prove that discrete exponentials $f_{?}(z)$ for such $z$ that

$$
f_{b+1}(z)=-f_{b}(z)
$$

precisely form a basis of discrete holomorphic functions on $\Gamma$ tiling $A_{q}$ twisted by the antiperiodic spin structure on $[0,1], q=2 \pi i \tau$. One precise statement (for the case of a rhomboid graph) is as follows:

Proposition 12. Consider a bipartite graph $\Gamma$ critically embedded into the torus $E_{\tau}=$ $\mathbb{C} /\langle 1, \tau\rangle(\operatorname{Im}(\tau)>0)$ in such a way that there exist complex numbers $a_{1}, \ldots, a_{2 n}, b_{1}, \ldots, b_{2 m}$ of equal absolute value such that $a_{1}+\ldots+a_{2 n}=1, b_{1}+\ldots+b_{2 m}=\tau$, and the corresponding rhomboid graph on $\Gamma \amalg \Gamma^{*}$ has edges

$$
\begin{gathered}
\left(a_{1}+\ldots+a_{k}+b_{1}+\ldots+b_{\ell}, a_{1}+\ldots+a_{k+1}+b_{1}+\ldots+b_{\ell}\right), \\
\left(a_{1}+\ldots+a_{k}+b_{1}+\ldots+b_{\ell}, a_{1}+\ldots+a_{k}+b_{1}+\ldots+b_{\ell+1}\right)
\end{gathered}
$$

(here we consider the subscripts as elements of $\mathbb{Z} / m, \mathbb{Z} / n$ ). Assume further that the vertex 0 is black, and assume that the spin structure of $E_{\tau}$ around $[0,1]$ is antiperiodic. Then the Kasteleyn matrix of $\Gamma$ satisfies

$$
p f\left(K_{\Gamma}\right)=2^{m} \prod_{j=1}^{n}\left(1 \mp f_{\tau}\left(z_{j}\right)\right)
$$

where $f_{b}(z)$ are Kenyon exponentials [21], and $z_{j}$ ranges over all complex numbers for which

$$
f_{1}\left(z_{j}\right)=-1,
$$

and the sign depends on the spin structure of $[0, \tau]$.

Remark: This is not as easy to generalize as one may think. In particular, in case of more general Riemann surfaces, a straightforward generalization of this formula is false. A heuristic explanation may be extracted from Section 4 above: In general, we are unable to identify functions with $1 / 2$-forms in boundary behavior, and transformation rules for $1 / 2$-forms would have to be modelled discretely. 
Proof: Because of continuity, it suffices to prove the formula for generic values of $a_{i}, b_{j}$. Consider for $k=0, \ldots, m-1$ the sets of black vertices of $\Gamma$

$$
B_{k}=\left\{b_{1}+\ldots+b_{2 k}+a_{1}+\ldots+a+2 i \mid i=0, \ldots, n-1\right\} .
$$

and the sets of white vertices of $\Gamma$

$$
W_{k}=\left\{b_{1}+\ldots+b_{2 k+1}+a_{1}+\ldots a_{2 i+1} \mid i=0, \ldots, n-1\right\} .
$$

The Kenyon exponential function $f_{b}(z)$ with base point 0 is on the black vertex

$$
b=a_{1}+\ldots+a_{2 \ell}+b_{1}+\ldots+b_{2 k}
$$

equal to

$$
\prod_{i=1}^{k} \frac{1+z b_{2 i-1}}{1-z b_{2 i}} \prod_{j=1}^{\ell} \frac{1+z a_{2 j-1}}{1-z a_{2 j}} .
$$

Recall that we denoted by $z_{1}, \ldots, z_{n}$ all numbers satisfying (29). Consider functions

$$
f_{?, k}\left(z_{j}\right): B \rightarrow \mathbb{C}
$$

where

$$
\begin{aligned}
& f_{b, k}\left(z_{j}\right)=f_{b}\left(z_{j}\right) \text { when } b \in B_{k} \text {, } \\
& =0 \quad \text { when } b \notin B_{k} \text {. }
\end{aligned}
$$

We claim that for generic values of $a_{i}, b_{j}, f_{?, k}\left(z_{j}\right)$ form a basis of the space of all functions $B \rightarrow \mathbb{C}$. In effect, it is true for $a_{i}=\frac{1}{2 n}$ by direct computation, and hence it is true generically (since non-degeneracy occurs on a Zariski-open set).

Now by direct computation,

$$
\begin{aligned}
& \bar{\partial} f_{?, k}\left(z_{j}\right)=g_{?, k}\left(z_{j}\right)-g_{?, k-1}\left(z_{j}\right) \text { for } k=1, \ldots, m-1 \\
& \bar{\partial} f_{?, 0}\left(z_{j}\right)=g_{?, 0}\left(z_{j}\right) \mp f_{\tau}\left(z_{j}\right) g_{?, n}\left(z_{j}\right)
\end{aligned}
$$

where

$$
g_{?, k}\left(z_{j}\right): W \rightarrow \mathbb{C}
$$

are functions defined as follows:

$$
\begin{aligned}
& g_{b_{1}+\ldots+b_{2 \ell+1}+a_{1}+\ldots+a_{2 i+1}, k}\left(z_{j}\right):= \\
& f_{b_{1}+\ldots+b_{2 \ell}+a_{1}+\ldots+a_{2 i+2}, k}\left(z_{j}\right)-f_{b_{1}+\ldots+b_{2 \ell}+a_{1}+\ldots+a_{2 i}, k}\left(z_{j}\right) .
\end{aligned}
$$

Note that for each $j$, the matrix (30) in the basis $\left(f_{?}, k\left(z_{j}\right), g_{?, k}\left(z_{j}\right)\right)$ is

$$
\left(\begin{array}{crrrrr}
1 & -1 & & & & \\
& 1 & -1 & & & \\
& & 1 & -1 & & \\
\ldots & \cdots & \cdots & \cdots & \cdots & \cdots \\
\mp f_{\tau}\left(z_{j}\right) & & & & & 1
\end{array}\right),
$$

which has determinant

$$
1 \mp f_{\tau}\left(z_{j}\right) \text {. }
$$

But now the function $h_{?, k}: W \rightarrow \mathbb{C}$ which is defined by

$$
h_{b_{1}+\ldots+b_{2 \ell+1}+a_{1}+\ldots+a_{2 i+1}, k}\left(z_{j}\right):=f_{b_{1}+\ldots+b_{2 \ell}+a_{1}+\ldots+a_{2 i+2}, k}\left(z_{j}\right)
$$


plays a symmetric role to $f_{?, k}$ which respect to exchanging $\bar{\partial}$ for $\partial$, and the base change between $g$ and $h$ is conjugate to the matrix

$$
\left(\begin{array}{rrrrrr}
1 & -1 & & & & \\
& 1 & -1 & & & \\
& & 1 & -1 & & \\
\ldots & \ldots & \ldots & \ldots & \ldots & \ldots \\
1 & & & & & 1
\end{array}\right),
$$

which is 2 .

To try to obtain a limit over tilings with decreasing length of rhomboid edge, it further seems natural to use a recipe of Mercat [26] how to take the limit in order to make discrete exponentials converge to ordinary continuous exponentials: subdivide each rhomboid into 4 equal rhomboids with parallel edges, and repeat this procedure. Even in simple examples, however, we see that this procedure when applied to (26), will not produce a convergent limit in the naive sense: the limit has to be "regularized" (which is not surprising, given the usual physical context of lattice regularization). For example, if the segment $[0,1]$ is tiled into equal segments by the edges of $\Gamma$, the equation (27) becomes

$$
\left(\frac{1-z / N}{1+z / N}\right)^{2 N}=-1,
$$

which implies that $z$ is on the imaginary line. Then (26) becomes a product of quantities of the form

$$
1 \pm e^{-z \tau},
$$

so given $\operatorname{Im}(\tau)>0$, for $\operatorname{Im}(z)>0$, the second summand (32) will have absolute value $>1$. The number of such terms will increase with $N$, as solutions of (27) in increasing bounded regions will approach $(2 k+1) \pi i, k \in \mathbb{Z}$. Therefore, the expression (26) in this case cannot converge as $N \rightarrow \infty$. The "limit" has to be taken in the sense of

$$
\begin{gathered}
\prod_{n \in \mathbb{Z}}\left(1-q^{n+\frac{1}{2}}\right)= \pm \prod_{n \in \mathbb{N}}\left(1-q^{n+\frac{1}{2}}\right)^{2} q^{-\frac{1}{2}-\frac{3}{2}-\frac{5}{2}-\cdots} \sim \\
\sim \prod_{n \in \mathbb{N}}\left(1-q^{n+\frac{1}{2}}\right)^{2} q^{-\frac{1}{24}},
\end{gathered}
$$

(the right hand side being the desired ratio of a theta function by $\eta$ ), based on the "calculation"

$$
\begin{gathered}
-\frac{1}{2}-\frac{3}{2}-\frac{5}{2}-\ldots \sim \\
\sim\left(-\frac{1}{2}-1-\frac{3}{2}-2-\frac{5}{2}-\ldots\right)+(1+2+3+\ldots) \sim-\frac{1}{2} \zeta(-1)+\zeta(-1)=-\frac{1}{24} .
\end{gathered}
$$

In this special case, the regularization can be achieved by multiplying (26) by

$$
\prod_{\operatorname{Im}(z)>0} \prod \frac{1}{f_{b+\tau}(z)}
$$


where the product is over solutions of (27) with $\operatorname{Im}(z)>0$. This regularization indeed produces the desired ratio of $\theta$ and $\eta$ in the limit, multiplied by the factor $q^{1 / 24}$.

Therefore, in some sense, for a general graph, the question entails finding a regularization procedure which would generalize (33). Again, one precise statement can be obtained as follows. Define

$$
: p d\left(K_{\Gamma}\right):=\prod_{j=1}^{n}\left(1-f_{\tau}\left(i\left|z_{j}\right|\right)\right)
$$

Proposition 13. Suppose graphs $\Gamma_{k}$ are as in Proposition 12, and that the rhomboid graph of $\Gamma_{k+1}$ is obtained from the rhomboid graph of $\Gamma_{k}$ by subdividing each rhomboid into 4 congruent rhomboids with parallel edges. Then

$$
\lim _{k \rightarrow \infty}: p f\left(K_{\Gamma_{k}}\right):=\prod_{j=0}^{\infty}\left(1 \mp q^{j+\frac{1}{2}}\right)^{2} .
$$

Proof: We must study solutions $z_{j}$ of the equation

$$
\prod_{i=1}^{n} \frac{\left(1+\frac{a_{2 i-1} z}{N}\right)^{N}}{\left(1-\frac{a_{2 i} z}{N}\right)^{N}}=-1 .
$$

We rewrite this as

$$
\sum_{i=1}^{n}\left(\ln \left(1+\frac{a_{2 i-1} z}{N}\right)-\ln \left(1-\frac{a_{2 i} z}{N}\right)\right)=\frac{2 k+1}{N} \pi i, k \in \mathbb{Z} .
$$

Put

$$
f(t):=\sum_{i=1}^{n}\left(\ln \left(1+a_{2 i-1} t\right)-\ln \left(1-a_{2 i} t\right)\right)
$$

Then

$$
f(0)=0
$$

and $f$ is analytic in a neighborhood of 0 with non-zero derivative at 0 . Hence, the same holds for the inverse $g=f^{-1}$. From this, (substituting $t=\frac{z}{N}$ ), we have the following

Lemma 14. For every $\delta>0$ there exists a $c>0$ such that for every $N$, when

$$
|z|<c N
$$

then $\frac{z}{N}$ is in the domain of $g$, and all the solutions to (34) satisfying (35) are of the form

$$
z_{k}=(2 k+1) \pi i+\epsilon_{k},\left|\epsilon_{k}\right|<\frac{\delta k}{N} .
$$


Now consider an inequality of the form

$$
C^{-1 / M}<\left|\frac{1-q^{|z| / 2 \pi}}{1-q^{|k+1 / 2|}}\right|<C^{1 / M}
$$

where $C>1$ is some constant. (37) holds when

or

$$
|\ln | 1-q^{|z| / 2 \pi}|-\ln | 1-q^{|k+1 / 2|}||<\frac{\ln C}{M}
$$

$$
\left|q^{|z| / 2 \pi}-q^{|k+1 / 2|}\right|<\frac{\ln C}{2 M}
$$

This follows whenever

$$
\left|\frac{|z|}{2 \pi}-\left(\left|k+\frac{1}{2}\right|\right)\right|<\frac{k}{M}
$$

for some constant $K>0$ dependent on $C$, since $q^{t}$ has a bounded derivative in $t>0$ for $0<|q|<1$.

Now set $M:=\sqrt{N}$. By the Lemma, there exist constants $A, B>0$, (we may assume $A<1$ ) such that (38) (and hence (37)) holds for some solution $z_{j}$ whenever

$$
|k|<A \sqrt{N},
$$

and

$$
\left|z_{j}\right|>B \sqrt{N} \text { for all } j \in S
$$

where $S$ is the set of all other indices $j$. Now note that

$$
\prod_{j \in S}\left(1-q^{\left|z_{j}\right| / 2 \pi}\right)=O\left(\sum_{j \in S}\left(\left|q^{\left|z_{j}\right| / 2 \pi}\right|\right)=O\left(N|q|^{B \sqrt{N} / 2 \pi}\right) .\right.
$$

The limsup of the right hand side of (41) with $N \rightarrow \infty$ is 0 . Thus, the solutions (40) may be neglected. Also, the quantity

$$
\prod_{k>A \sqrt{N}}\left(1-q^{|k+1 / 2|}\right)^{2}
$$

approaches 0 as $N \rightarrow \infty$, and by (37),

$$
\frac{1}{C}<\prod_{|k|<A \sqrt{N}}\left|\frac{1-q^{|z| / 2 \pi}}{1-q^{(|k+1 / 2|)}}\right|<C,
$$

as $M=\sqrt{N}$ and $A<1$. Since $C>1$ was an arbitrary constant, we are done. 


\section{Fermions on a Riemann surface II: A BRIEF Review of CONFormal field THEORY, WITH SPECULATIONS ABOUT GRAPHS}

In physics, it is "known" that the critical Ising model on a lattice converges to the 1dimensional free fermion theory [29], cf. [7], while the critical dimer model converges to the free fermion of space-time dimension 2 ([8]). To obtain rigorous mathematical theorems in these directions, we need to take into account the detailed structure of the fermionic CFT's, and model it in graph theory. Such mathematical treatments of conformal field theory now exist, (cf. [31, 22] from the Segal point of view, [9] from the vertex algebra point of view), but the structure involved is somewhat complicated. Because of this, we take time in this section to survery these structures in more detail. Some comments on their potential impact on the graph theory side of the story will be made at this section's conclusion. For simplicity, throughout this section, we will consider complex CFT structures, as real structures complicate things further.

A big part of the story of fermion CFT's is bosonisation. For a bosonic CFT, we are supposed to have a real-analytic line bundle $L$ on the moduli space of Riemann surfaces $\Sigma$ with analytically parametrized boundary, a complex Hilbert space $H$ and, writing $H_{\partial \Sigma}$ for

$$
H^{* \hat{\otimes} m} \hat{\otimes} H^{\hat{\otimes} n}
$$

when $\Sigma$ has $m$ inbound and $n$ outbound boundary components (we take $\mathbb{C}$ when $\Sigma$ is closed), a map

$$
L_{\Sigma} \rightarrow H_{\partial \Sigma}
$$

satisfying gluing axioms analogous to Lemma 11 (cf. [31, 18, 11]).

A genus $g$ Riemann surface $\Sigma_{g}$ can be cut along $g$ non-separating disjoint real-analytic simple curves $a_{1}, \ldots, a_{g}$ into a genus 0 surface $A$ with $2 g$ boundary components (let, for future reference, a "dual cutting" by real-analytic simple curves $b_{1}, \ldots, b_{g}$.). Gluing a standard disk on each of these boundary components, we obtain a sphere. Since the 2-sphere possesses a unique conformal structure, this specifies an element

$$
\iota_{a_{1}, \ldots, a_{g}} \in L_{\Sigma_{g}}
$$

hence, by (42), a complex number. This assignment

$$
A \mapsto Z\left(a_{1}, \ldots, a_{g}\right) \in \mathbb{C}
$$

is called the genus $g$ partition function. For $g=1$, one can take the standard annulus $A_{q} \subset \mathbb{C}$ with boundary components $S^{1}$ and $q S^{1}$ parametrized by the functions $z, q z$, respectively, which gives us the partition function $Z(q)$. This function is a series in

$$
q^{a} \bar{q}^{b}
$$

$(0<\|q\|<1)$ with positive integral coefficients where $a-b \in \mathbb{Z}$, and the coefficient of (44) counts the number of states of $H$ of weight $(a, b)$. In other words, it is the trace of the "grading operator" which, at weight $(a, b)$, is given by multiplication by $q^{a} \bar{q}^{b}$.

There is a special class of CFT's called rational conformal field theory, abbr. RCFT. (One rigorous mathematical approach was reached by Huang and Lepowsky [19], although it doesn't use the language of Hilbert spaces, but the structure of vertex algebra. An approach 
via Hilbert spaces was developed in [18, 11]. To date, the two approaches have not been fully unified, and each has certain desirable features.) In this case, we have a finite set $\Lambda$ of sectors with a distinguished element $0 \in \Lambda$ and an involution (?)* $\Lambda \rightarrow \Lambda, 0^{*}=0$, and decompositions

$$
\begin{gathered}
H=\bigotimes_{\lambda \in \Lambda} H_{\lambda} \hat{\otimes} \bar{H}_{\lambda^{*}}, \\
Z(q)=\sum_{\lambda \in \Lambda} Z_{\lambda}(q) Z_{\lambda^{*}}(\bar{q}) .
\end{gathered}
$$

(Sometimes, elements of $\Lambda$ are referred to as labels, an indexing set for sectors. We choose here to identify each label with its corresponding sector.) The precise explanation of (45) is that we have certain line bundles $M_{\lambda}$ on the moduli space of all annuli, and a positive-definite Hermitian pairing

$$
\bigoplus_{\lambda \in \Lambda} M_{\lambda} \otimes \bar{M}_{\lambda^{*}} \rightarrow L
$$

and we have

$$
Z_{\lambda}(q): M_{\lambda} A_{q} \rightarrow \mathbb{C}
$$

so (45) makes sense.

This is actually the tip of an iceberg called chiral conformal field theory: One is required to have a finite-dimensional complex vector space $M_{\lambda} \Sigma$ for each Riemann surface $\Sigma_{\left(\lambda_{i}\right)}$ with parametrized boundary components labeled by the $\lambda_{i}$ 's, which are supposed to satisfy both (46) and gluing axioms [18, 11]. The most important part of the gluing structure is an isomorphism

$$
M \check{\Sigma}_{\left(\lambda_{i}\right)} \cong \bigoplus_{\lambda \in \Lambda} M \Sigma_{\left(\lambda_{i}, \lambda, \lambda\right)}
$$

where $\check{\Sigma}$ is obtained from $\Sigma$ by gluing an inbound and an outbound boundary component: the sum on the right hand side is over all possible labellings of the two additional boundary components by the same label. Additionally, there are to be linear maps

$$
M \Sigma_{\left(\lambda_{i}\right)} \rightarrow H_{\partial \Sigma_{\left(\lambda_{i}\right)}}
$$

satisfying appropriate gluing axioms $([18,11])$. It is worth mentioning that for each $\lambda \in$ $\Lambda$, the line bundle $M_{\lambda} A$ specifies a holomorphic $\mathbb{C}^{\times}$-central extension of the semigroup of annuli with analytically parametrized boundary components of opposite orientations; these central extensions do not depend on $\lambda$, and are characterized by a single number called the central charge and denoted by $c$ - see [31]. (Note: there is an infinite-dimensional space of annuli with analytically parametrized boundary components modulo the relation of conformal equivalence preserving the boundary parametrizations; if we restricted only to the standard annuli $A_{q}$, we would get the semigroup $\mathbb{C}_{0<q<1}$ which does not have non-trivial central extensions.)

Models of the fermion CFT which satisfy the above axioms are referred to as bosonised fermions. From the point of view of string theory, a fermion of spacetime dimension $d$ has central charge $d / 2$. The theory differs depending on whether $d$ is odd or even. For $d=1$, 
the theory can be constructed, for example, as a coset model of the level 2 WZW-model for $S U(2)([7], 18.5 .1, k=2)$, or as a non-supersymmetric minimal model [7], 7.4.2.

Generally, for $d$ odd, the $d$-dimensional bosonised fermion theory has three sectors

$$
\Lambda=\left\{N S^{+}, N S^{-}, R\right\}, \lambda^{*}=\lambda, 0=N S^{+} .
$$

(R stands for Ramond and NS stands for Neveu-Schwarz.) Its partition function is determined by letting $Z_{N S^{+}}(q), Z_{N S^{-}}(q)$ be the integral (resp. integral $+1 / 2$ )-dimensional terms of

$$
\prod_{n \geq 0}\left(1+q^{n+\frac{1}{2}}\right)^{d}
$$

and

$$
Z_{R}(q)=2^{\lfloor d / 2\rfloor} \prod_{n>0}\left(1+q^{n}\right) .
$$

The power 2 in the formula for $Z_{R}(q)$ is significant, it expresses the fact that the bottom weight space of $H_{R}$ is naturally the spinor module of the complex Clifford algebra on a space of dimension $d$. The dimension of the modular functor on a Riemann surface of genus $g$ is

$$
\frac{1}{2}\left(4^{g}+2^{g}\right)
$$

which is equal to the number of spin structures of Arf invariant 0.

The bosonised fermion of even spacetime dimension $d$ has sector set

$$
\Lambda_{2}=\left\{N S^{+}, N S^{-}, R_{+}, R_{-}\right\} .
$$

We have

$$
R_{+}^{*}=R_{-} \text {. }
$$

Here the labels we chose express the operations on the sector Hilbert spaces of the 1dimensional fermion. In particular, $H_{R} \hat{\otimes} H_{R}$ decomposes into two state spaces, according to the decomposition of the complex spinor on $\mathbb{R}^{2}$. Its partition function is determined by letting $Z_{N S^{+}}(q), Z_{N S^{-}}(q)$ be the integral (resp. integral $+1 / 2$ )-dimensional terms of

$$
\prod_{n \geq 0}\left(1+q^{n+\frac{1}{2}}\right)^{d}
$$

and

$$
Z_{R_{ \pm}}(q)=2^{d / 2} \prod_{n>0}\left(1+q^{n}\right)
$$

The reason why there are now two Ramond sectors is that there are two non-isomorphic complex spinors in even dimension. The dimension of the modular functor on a Riemann surface of genus $g$ is

$$
4^{g}
$$

which is equal to the number of all spin structures.

For $d=2$, the bosonised fermion theory turns out to be isomorphic to the conformal field theory associated with the lattice $2 \mathbb{Z} \subset \mathbb{R}([12,18])$. This phenomenon is known as the boson-fermion correspondence. Expressed this way, the labels become

$$
\{0,1 / 2,1,3 / 2\},(1 / 2)^{*}=(3 / 2) .
$$


The chiral partition functions are

$$
Z_{\lambda}(q)=\sum_{k \in \mathbb{Z}} q^{\frac{1}{2}(k+\lambda)^{2}} \prod_{n>0}\left(1-q^{n}\right)^{-1} .
$$

We see that regardless of the parity of dimension, the partition function of the fermion of dimension $d$ is

$$
\begin{aligned}
& Z(q)=\frac{1}{2}\left(\prod_{n \geq 0}\left(1+q^{n+1 / 2}\right)\left(1+\bar{q}^{n+1 / 2}\right)+\prod_{n \geq 0}\left(1-q^{n-1 / 2}\right)\left(1-q^{n-1 / 2}\right)+\right. \\
& \left.2^{d+1}(q \bar{q})^{d / 16} \prod_{n \geq 1}\left(1+q^{n}\right)\left(1+\bar{q}^{n}\right)\right) .
\end{aligned}
$$

Note that for $d=2$, this is indeed equal to

$$
\begin{aligned}
& \left(\sum_{k \in \mathbb{Z}}\left((q \bar{q})^{2 k^{2}}+(q \bar{q})^{\frac{1}{2}(2 k+1)^{2}}+2(q \bar{q})^{\frac{1}{2}\left(2 k+\frac{1}{2}\right)^{2}}\right)\right) . \\
& \cdot \prod_{m, n>0}\left(1-q^{n}\right)^{-1}\left(1-\bar{q}^{n}\right)^{-1}
\end{aligned}
$$

which is what we obtain when we combine the chiral partition functions (51). Note that in (53) and (52), in fact the sums of the first two summands are equal by Jacobi's triple product identity, as are the last summands.

How is it possible that the dimensions of the modular functor (49) and (50) in the even and odd-dimensional case are different, even though the partition function (52) has a uniform expression? The answer is that in the even-dimensional case, when the spin structure along some of the curves $a_{i}$ is anti-periodic, the two Ramond sectors arising will have the same partition function, and hence their sum can be grouped in the power of 2 coefficient.

The genuinely fermionic approach to fermion RCFT uses another, more complicated, axiomatization. This time, the chiral parts are chiral super conformal field theories. (This is a different concept from a supersymmetric conformal field theory, where both a bosonic and a fermionic part are present.) Chiral super conformal field theories are axiomatized similarly as chiral conformal field theories, but the Riemann surfaces $\Sigma$ involved come with spin structure. We then have a decomposition

$$
\Lambda=\Lambda_{N S} \amalg \Lambda_{R},
$$

expressing which labels apply to antiperiodic and which to periodic boundary components. Additionally, we deal with super-modular functors, which means that each $M \Sigma_{\left(\lambda_{i}\right)}$ is a $\mathbb{Z} / 2$ graded vector space, as is each sector state space

$$
H_{\lambda} .
$$

The maps (48) are required to be graded, and the usual sign convention

$$
(-1)^{i j}
$$

is applied with interchanging elements of degree $i, j$. We now have chiral partition functions

$$
Z_{\lambda}^{N S}(q), Z_{\lambda}^{R}(q)
$$


where the superscript expresses the spin structure in the radial direction of the annulus. When factoring a CFT to fermionic chiral factors, the analog of formula (45) at both chiralities becomes

$$
Z(q)=\frac{1}{2}\left(\sum_{\lambda \in \Lambda} Z_{\lambda}^{N S}(q) Z_{\lambda^{*}}^{N S}(\bar{q})+\sum_{\lambda \in \Lambda} Z_{\lambda}^{R}(q) Z_{\lambda^{*}}^{R}(\bar{q})\right)
$$

For a Riemann surface of arbitrary genus $g$ where the summation is over spin structures on the Riemann surface $\Sigma$, and over a product of the sets of periodic (resp. antiperiodic) labels matching to the spin structure of each of the curves $a_{i}, b_{i}$, and the coefficient before the sum is $1 / 2^{g}$. (This can be thought of as an averaging over spin structures, or, in analogy with string theory, a "GSO projection".) When there is exactly one periodic and one antiperiodic label, one can show that the super-modular functor is always one-dimensional for each spin structure on a Riemann surface, and is invertible in the sense that its tensor product with another super-modular functor is the trivial (unit) super-modular functor. In this case, the summation is precisely over the set of spin structures on $\Sigma$. This, in fact, is the CFT explanation of the decomposition of the discrete partition functions of the Ising and dimer models into summands corresponding to spin structures, with signs given by the Arf invariant (see e.g. formula 2 above); those are the summands whose gluing we study in Sections 2 and 3. There is one substantial difference in the "CFT limit", however: one can show that the summands corresponding to spin structures of Arf invariant 1 are always equal to 0 . From a physics point of view, this can be justified by noting that the contribution of such terms would come with a negative sign, which is physically impossible. A mathematical argument can also be obtained along the lines of [22].

It is also possible to talk about a fermionic conformal field theory with both chiralities, which consists of two super-Hilbert spaces $H_{R}$ and $H_{N S}$ ( $R$ corresponds to the periodic and $N S$ to the anti-periodic spin structure on $S^{1}$ ), a real-analytic line bundle $L$ on the moduli space of Riemann surfaces with spin structure and with (analytically) parametrized boundary components. The conformal field theory then specifies, again, a map

$$
L_{\Sigma} \rightarrow H_{\partial \Sigma}
$$

where $H_{\partial \Sigma}$ is, again, a Hilbert tensor product of copies of $H_{R}, H_{N S}$ and their duals, depending on orientation and spin structure of each boundary components. The gluing axioms are analogous to the bosonic case (Note: no super-structure is present on $L$, or in other words, it is assumed to be "even".)

Given a fermionic chiral RCFT, the spaces $H_{R}$ and $H_{N S}$ are given by

$$
\bigoplus_{\lambda} H_{\lambda} \hat{\otimes} \bar{H}_{\lambda^{*}}
$$

where the sum is taken over R resp. NS labels. For a closed Riemann surface $\Sigma$, one then has one partition function for each spin structure, although the partition functions corresponding to spin structures of Arf invariant 1 are 0 . The sum of these fermionic partition functions, multiplied by the factor $1 / 2^{g}$, is the bosonic partition function (54). 
There is a $d$-dimensional chiral free fermion with invertible super-modular functor when $d$ is even. The chiral fermionic partition functions are

$$
\begin{gathered}
{ }_{c} Z_{N S}^{R}(q)=\prod_{n \geq 0}\left(1+q^{n+\frac{1}{2}}\right)^{d}, \\
{ }_{c} Z_{N S}^{N S}(q)=\prod_{n \geq 0}\left(1-q^{n+\frac{1}{2}}\right)^{d}, \\
{ }_{c} Z_{R}^{N S}(q)=2^{d / 2} \prod_{n>0}\left(1+q^{n}\right)^{d}, \\
{ }_{c} Z_{R}^{R}(q)=0
\end{gathered}
$$

(We use the symbol ${ }_{c} Z$ to distinguish the chiral partition functions from the fermionic partition functions at both chiralities, which are written below in (59), (60), (61), (62), since there is only one label for each spin structure.) The conformal field theory assembled from this super-RCFT is isomorphic to the conformal field theory of the bosonised $d$-dimensional fermion described above.

For $d$ odd, there is no d-dimensional chiral fermion super-conformal field theory as defined above. It was proved in 22 that there is no invertible super-modular functor of central charge 1/2. However, P.Deligne found an even more involved axiomatization using the super Brauer group and central simple algebras which does allow a 1-dimensional chiral fermion model. Roughly, the super-Brauer group of $\mathbb{C}$ is $\mathbb{Z} / 2$, and then non-zero element is represented by the super-division algebra $C=\mathbb{C}[a] /\left(a^{2}-1\right)$ where $a$ is odd. Then the value of the modular functor (and the Hilbert spaces) are taken in the category of modules over super-division algebras, and identification is allowed under super-Morita equivalence. When $d$ is odd, 1dimensional $C$-modules occur as values of the modular functor when Ramond boundary components arise.

When we put both chiralities together, there is, again, a uniform formula for the fermionic (spin-structure dependent) partition functions of the $d$-dimensional fermion, regardless of the parity of $d$ :

$$
\begin{gathered}
Z_{N S}^{R}(q)=\prod_{n \geq 0}\left(1+q^{n+\frac{1}{2}}\right)^{d}\left(1+\bar{q}^{n+\frac{1}{2}}\right)^{d} \\
Z_{N S}^{N S}(q)=\prod_{n \geq 0}\left(1-q^{n+\frac{1}{2}}\right)^{d}\left(1-\bar{q}^{n+\frac{1}{2}}\right)^{d} \\
Z_{R}^{N S}(q)=2^{d} \prod_{n>0}\left(1+q^{n}\right)^{d}\left(1+\bar{q}^{n}\right)^{d} \\
Z_{R}^{R}(q)=0
\end{gathered}
$$

In fact, as a fermionic conformal field theory (i.e. on spin structure at a time), the $d$ dimensional fermion is a tensor product of $d$ copies of the 1-dimensional fermion (and its rigorous mathematical construction in fact proceeds in this fashion). 
For $d$ odd, the Hermitian form

$$
C \otimes \bar{C} \rightarrow \mathbb{C}
$$

has

$$
\langle 1,1\rangle=\langle a, a\rangle=1,
$$

which causes, on the $\mathrm{R}$ label part of the modular functor, which is given by Deligne's prescription in the form of a (1-dimensional) graded $C$-module, the additional factor 2, explaining the odd power of 2 present in (61).

Comments and questions about graphs: Physics [8] predicts that there should exist a limit formula from the critical dimer model to the 2-dimensional free fermion conformal field theory, i.e. the theory which has a bosonic description with modular functor with set of labels $\Lambda_{2}$, as well as a fully fermionic description with an invertible super-modular functor.

On the other hand, physics also predicts [29] that there should exist a limit formula from the critical Ising model to the bosonic 1-dimensional fermion theory with the set of labels $\Lambda$. We saw that both conformal field theories are closely related yet substantially different.

The factorization formulas (45) and (54), and their higher genus analogues, suggest compelling analogies with decompositions of partition functions of the discrete models into summands. This is particularly striking in view of the expression of the dimer and Ising model partition functions as a sum of graphs drawn in a suitable way on genus $g$ Riemann surfaces as sums of $4^{g}$ Pfaffians. In fact, an important point of this paper is that in Sections 2 and 3. we have modeled discrete gluing analogues for the fermionic form of the conformal field theory, i.e. (59), (60), (61), (62).

It is not clear whether (or in what situations) the estimates for number of summands given by the limit theory are precise: In the conjectured limit, in the fermionic description, (62), and more generally, the partition functions corresponding to spin structures of Arf invariant 1, always vanish (see also [8]). This may appear to contradict the result of Norin [27] showing that for $g=1$, a 3-Pfaffian graph is always 1-Pfaffian, while the limit formula predicts 3 non-vanishing factors. However, Norin's result [27] is stated in a form not involving weights of edges. When translated to our formulation with weights, cancellations may occur, and some terms may vanish. This occurs specifically in the case of critical embeddings, even without taking the limit.

Note that in the bosonic description, the interpretation of the limit is somewhat different. In this case, the summands are not Pfaffians, but suitable averages of Pfaffians using the GSO projection. In this case, in the even-dimensional case, none of the summands actually vanish, but subsets of different summands have identical partition functions: in the discrete case, then, the question of vanishing is replaced by other linear dependencies.

In the odd-dimensional case (which is relevant to the Ising model), the situation is more interesting: In the bosonic description, one doesn't combine sectors with the same partition function, but instead the sectors themselves combine to one sector. Is there a definition of corresponding "GSO-projected" summands purely in terms of graphs?

Alternately, in the fermionic description, is there a graph-theoretic characterization of Pfaffians corresponding to the modules over the Deligne super-algebra $C$ ? While an expression of the Ising partition function as a sum of $4^{g}$ Pfaffians is obtained in [24], this description uses a modifying construction on the underlying graph. Is there a formula in 
terms purely of Pfaffian-like summands on the original graph, suitably generalized using $C$-modules discretely mimicking Deligne's theory?

\section{REFERENCES}

[1] L. Alvarez-Gaume, G. Moore, C. Vafa: Theta functions, modular invariance, and strings, Comm. Math. Phys. Volume 106, Number 1 (1986), 1-40.

[2] A. A. Beilinson, V. Drinfeld: Chiral algebras, ISBN-10: 0-8218-3528-9, AMS Colloquium Publications, vol. 51.

[3] R. E. Borcherds: Monstrous Moonshine and Monstrous Lie Superalgebras, Invent. Math. 109, 405444, 1992.

[4] Cimasoni, D., Reshetikhin, N.: Dimers on surface graphs and spin structures, Communications in Mathematical Physics, Vol. 275, 2007, p. 187-208.

[5] Cimasoni, D., Reshetikhin, N.: Dimers on surface graphs and spin structures II., Communications in Mathematical Physics, Vol. 281, No. 2, 2009, p. 445-468.

[6] H.Cohn, R. Kenyon, J.Propp: A variational principle for domino tilings, J. Amer. Math. Soc. 14 (2001) 297-346

[7] P. Di Francesco, P. Mathieu, and D. Sénéchal: Conformal Field Theory, Springer-Verlag, New York, 1997. ISBN 0-387-94785-X.

[8] R. Dijkgraaf, D. Orlando, S. Reffert: Dimer models, free fermions and super quantum mechanics, Adv. Theor. Math. Phys. Volume 13, Number 5 (2009), 1255-1315.

[9] A.Feingold, F.X.Ries, M.D.Weiner: Spinor construction of the $c=1 / 2$ minimal model, in: Moonshine, the Monster and Related Topics, Contemporary Math. 193 (C.Dong and G. Mason, eds.), AMS, Providence, R.I., 1995, 45-92

[10] J.Ferdinand: Statistical mechanics of dimers on a quadratic lattice, J.Math. Phys. 8 (1967), 2332-2339

[11] T.Fiore, P.Hu and I.Kriz: Laplaza sets, or How to Select Coherence Diagrams for Pseudo Algebras, Advances in Mathematics 218 (2008) 1705-1722.

[12] T.Fiore, I.Kriz: What is the Jacobian of a Riemann surface with boundary?, Deformation spaces, 5374, Aspects Math., E40, Vieweg + Teubner, Wiesbaden, 2010

[13] M. E. Fischer: On the dimer solution of planar Ising problems, Journal of Mathematical Physics 710 (1966).

[14] I. Frenkel, J. Lepowski, and A. Meurman: Vertex operator algebras and the monster, Academic Press, Boston, 1988.

[15] A. Galluccio, M. Loebl: A Theory of Pfaffian Orientations I., Electronic Journal of Combinatorics 6,1 1999.

[16] A. Hassell, Analytic surgery and analytic torsion, Comm. in Anal. and Geom. 6, 255-289 (1998).

[17] R.Hortsch, I.Kriz, A.Pultr: A universal approach to vertex algebras, J. Algebra. 324 (2010), 1731-1753.

[18] P. Hu, I. Kriz: Conformal field theory and elliptic cohomology, Advances in Mathematics, Volume 189, Issue 2, 20 December 2004, Pages 325-412.

[19] Y.-Z. Huang, J. Lepowski: A theory of tensor products for module categories for a vertex operator algebra. I, II., Selecta Math. (N.S.) 1 (1995), no. 4, 699756, 757786.

[20] P. W. Kasteleyn, Dimer statistics and phase transitions. J. Math. Phys. 4 (1963), 287293.

[21] R. Kenyon: The Laplacian and Dirac operators on critical planar graphs, Invent. Math., 150 (2002), pp. 409439.

[22] I. Kriz: On Spin and modularity in conformal field theory, Ann. Sci. de ENS 36 (4) (2003), 1, 57112.

[23] I.Kriz, Y.Xiu: Tree field algebras: An algebraic axiomatization of intertwining vertex operators, arXiv:1102.2007

[24] M. Loebl, G. Masbaum, On the optimality of the Arf invariant formula for the graph polynomials, Advances in Mathematics Volume 226, Issue 1, 15 January 2011, Pages 332-349.

[25] M. Loebl, P. Somberg, Discrete Dirac Operators, Critical Embeddings and Ihara-Selberg Functions, http://xxx.lanl.gov/abs/0912.3200. 
[26] C. Mercat: Exponentials form a basis of discrete holomorphic functions, arXiv: math-ph/0210016.

[27] S. Norin: Drawing 4-Pfaffian graphs on the torus, Combinatorica 29 (2009), 109-119.

[28] A.A.A.Miranda, C.L.Lucchesi: Matching signatures and Pfaffian graphs, Discrete Mathematics, 2011, 289-294.

[29] L. Onsager: Crystal statistics. I. A two-dimensional model with an order-disorder transition, 1944, Phys. Rev. (2) 65 (34), 117149.

[30] A. N. Pressley and G. B. Segal: Loop groups, Oxford mathematical monographs, 1988, 318 pages.

[31] G. Segal: The definition of conformal field theory, in: Differential geometrical methods in theoretical physics (Como, 1987), NATO Adv. Sci. Inst. Ser. C Math. Phys. Sci., 250, Kluwer Acad. Publ., Dordrecht, 1988, 165-171.

[32] G. Tesler: Matching in graphs on non-orientable surfaces, J. Comb. Theory B 78 (2000), 198231.

[33] B. L. van der Warden: Die lange Reichweite der regelmassigen Atomanordnung in Mischkristallen, Z. Physik 118:473, (1941).

[34] E. Witten: Free fermions on an algebraic curve, The Mathematical Heritage of Hermann Weyl (Durham, NC, 1987), Proc. Sympos. Pure Math., vol. 48, Amer. Math. Soc., Providence, 1988, pp. 329344.

Igor Kriz, Department of Mathematics,, University of Michigan 2074 East Hall, 530 Church Street, Ann Arbor, MI 48109-1043, U.S.A.

E-mail address: ikriz@umich.edu

Martin Loebl, Dept. of Applied Mathematics and, Institute of Theoretical Computer Science (ITi), Charles University, Malostranske n. 25, 11800 Praha 1, Czech Republic.

E-mail address: loebl@kam.mff.cuni.cz

Petr Somberg, Mathematical Institute, Charles University, Sokolovska 83, 18000 Praha

8, Czech Republic.

E-mail address: somberg@karlin.mff.cuni.cz 